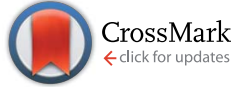

Cite this: Soft Matter, 2015, 11, 732

Received 18th October 2014 Accepted 26th November 2014

DOI: $10.1039 / \mathrm{c} 4 \mathrm{sm} 02294 \mathrm{~g}$

www.rsc.org/softmatter

\section{Topological energy storage of work generated by nanomotors}

\begin{abstract}
Fabian Weysser, ${ }^{a}$ Olivier Benzerara, ${ }^{a}$ Albert Johner ${ }^{\mathrm{ab}}$ and Igor M. Kulić ${ }^{\star a}$
Most macroscopic machines rely on wheels and gears. Yet, rigid gears are entirely impractical on the nanoscale. Here we propose a more useful method to couple any rotary engine to any other mechanical elements on the nano- and micro-scale. We argue that a rotary molecular motor attached to an entangled polymer energy storage unit, which together form what we call the "tanglotron" device, is a viable concept that can be experimentally implemented. We derive the torque-entanglement relationship for a tanglotron (its "equation of state") and show that it can be understood by simple statistical mechanics arguments. We find that a typical entanglement at low packing density costs around $6 k T$. In the high entanglement regime, the free energy diverges logarithmically close to a maximal geometric packing density. We outline several promising applications of the tanglotron idea and conclude that the transmission, storage and back-conversion of topological entanglement energy are not only physically feasible but also practical for a number of reasons.
\end{abstract}

\section{Introduction}

Molecular machines are everywhere around us and run our lives on the sub-cellular scale. Throughout evolution, Nature has come up with many practical and robust nano-machine designs, from DNA copying and protein producing machines to muscle driving nano-scale walkers. To mimic Nature and maybe even outperform it in some tasks seems to be humanity's undeclared goal in this century. What was science-fiction a decade ago takes shape in the physical reality as we speak. However, building functional, task performing nano-assemblies with comparable features as macro-scale machines still meets large obstacles. In particular, at the present stage it is virtually impossible to apply our machine building intuition gathered on the macroscopic scale. Interlocked motors with gears and wheels, connected with force transmitting chains and belts form the practical basis for most large scale mechanical devices. And yet they appear like a very awkward, hard to realize way to build nano-machines in the fluctuating realm of the soft and small.

While the motif of a classical rotary wheel is rare in Nature, ${ }^{1}$ it is occasionally utilized in some branches of life. For instance, bacteria's rotary flagellar engines enable them to move through their fluid environments. However, to benefit from their flagellar rotor the bacteria had to come up with a clever and efficient way to couple it to their environment. In their case, the task is fulfilled by a sophisticated filamentous helical coil - the

${ }^{a}$ Institut Charles Sadron, CNRS-UdS, 23 Rue du Loess, BP 84047, 67034 Strasbourg Cedex 2, France. E-mail: kulic@unistra.fr

${ }^{b}$ Department of Physics, Sejong University, Seoul 143-743, South Korea flagellum. The latter acts as a screw propeller, transmitting the motor rotation into translation and propulsion through the surrounding viscous fluid.

Thus, we could conclude that the problem in utilizing wheels, gears and rotors on the nano-scale in Nature is not the rotary engine mechanism itself. The difficulty lies rather in establishing an efficient and flexible transmission mechanism of the rotary movement to its surroundings.

In this report, we propose such a transmission mechanism that could be beneficial on the nano-scale. In particular, we will discuss a simple, generic way to implement a coupling of virtually any existing rotary nano- and microengines to their surroundings. The central idea that we want to advance is that of torque transmission by the process of topological entanglement of polymer chains attached to the active rotary device (motor), $c f$., Fig. 1. Such a motor equipped with a topological energy storage device we will in the following - alluding to the idea of entanglement - simply call the "tanglotron".

The concept of the tanglotron is related to the previously proposed idea of molecular motors operating in closed ring geometries ${ }^{2}$ combined with the natural idea of attaching polymer side chains for topologically trapping and preserving motor rotations. Numerous rotary engines exist in Nature ${ }^{3}$ with the most prominent examples being ATP-synthase, ${ }^{\mathbf{4 , 5}}$ the flagellar motor of bacteria ${ }^{6}$ and various DNA-topoisomerases. ${ }^{7}$ Several artificial directed rotors based on various operational principles have been developed..$^{3,8-11}$ New rotary engine designs continue to be conceptually proposed and still light up our imagination in nano-engineering. .,12 $^{2}$

The circularly rotating geometry of these numerous nanoengines is particularly suitable for generating entanglements 


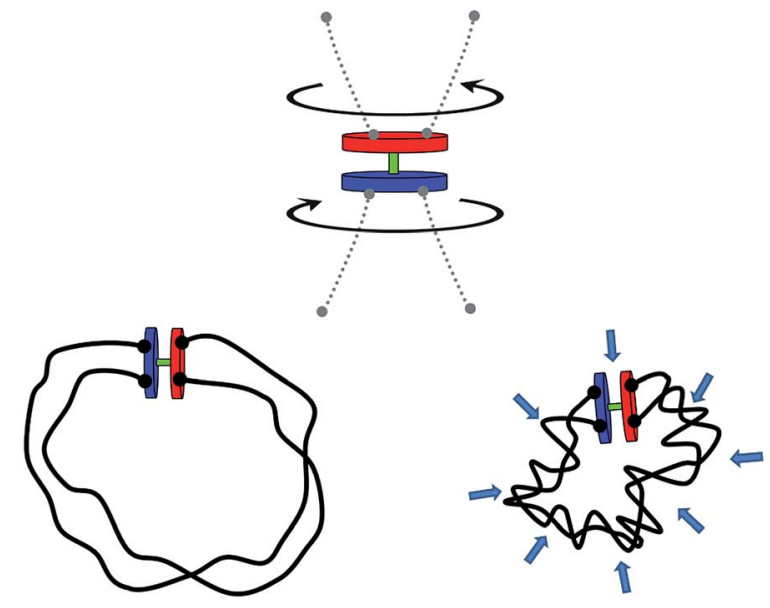

Fig. 1 The principle of a tanglotron. A rotary motor is coupled with two closed polymer loops. The rotation of the motor unit is trapped and conserved in the Gaussian entanglement of the polymer chains. The entangled chains collapse with increasing linking number generating a volumetric contraction.

and contraction in gel like materials. The effect of entanglement on the visco-elastic behavior of polymer gels and networks is a classical problem in polymer science. ${ }^{13-15}$ In a typical polymer melt, passively responding to sudden external strains, polymer entanglements are created and destroyed in a dynamic fashion, by the process of polymer "reptation". It is well-established that during mechanical shearing of a dense melt of long polymers, the relaxation of transient polymer entanglements is also the main contribution to dynamic stress relaxation. ${ }^{13-15}$ In this sense the concept of dynamic, transient entanglements is at the core of polymer physics.

The problems of fixed (permanent) topological entanglements have also attracted much theoretical interest. Simplified two dimensional models for the entanglements of flexible polymer chains have been developed in the past ${ }^{16-19}$ (reviewed in ref. 20) as well as more realistic but analytically less tractable 3 dimensional models have been considered..$^{21,22}$ Motivated by single DNA molecule probing experiments ${ }^{23}$ applying torques on the molecular scale by magnetic tweezers, the problem of DNA supercoiling, ${ }^{24}$ DNA braiding ${ }^{26}$ and its untwisting ${ }^{25}$ has been studied theoretically.

The idea of actively utilizing gel's permanent topology to tune and tweak its mechanical properties reaches quite far into the past. "Topological gels" containing sliding link pulley-like mechanisms were envisioned theoretically, ${ }^{13,27}$ long before their experimental manufacturing (chemical synthesis) could be realized in practice. ${ }^{28}$

Since the advent of DNA based life, living organisms naturally utilize the principle of topological energy storage. ${ }^{7,29,30}$ In particular, DNA double strand twisting and supercoiling are known to energetically shift the threshold for DNA opening and gene transcription as well as to provide an additional energy source for genome folding and compaction in prokaryotic organisms like bacteria. Besides DNA, other natural filaments, like single actin rings, are known to display similar supercoiling behavior. ${ }^{31}$ Beyond its obvious usefulness in natural processes, DNA supercoiling has also been suggested as a mechanism for processing information and performing simple computational tasks. $^{32}$

In this article opening the topic, we will focus on some central physical features associated with the tanglotron. In particular, we will study the problem of energy storage in the form of topological polymer-entanglements. We will derive the "equation of state" of the tanglotron and show that its torqueentanglement relationship can be understood by simple geometrical and statistical mechanics arguments. We conclude the article by outlining several benefits of actually building a tanglotron device, including possible applications from artificial muscles to nano-actuators for DNA nano-assemblies.

\section{Topological battery: storage of entanglement energy}

Let us consider a synthetic system shown in Fig. 1, consisting of an active rotary motor unit and two chains connecting the stator and the rotor of the device. In this section, we will first focus on the conceptual questions resulting from Fig. 1. These will be:

When the motor unit operates by $n$ turns how much energy is stored in its polymer chains?

How many topological entanglements (links) can be injected at a given maximal motor torque?

By how much do the chains of length $L$ collapse when loaded with $n$ entanglements?

Is there an upper topological storage capacity of the chains and how many topological links do fit inside for a given chain length $L$ ?

\section{A. Tanglotron simulated}

To gain insight into the system shown in Fig. 1, we set up a molecular dynamics simulation implementing the motor unit (red) and the topological container consisting of two long flexible polymer chains. The monomers within the chains interact purely repulsively by a Lennard-Jones short-range excluded volume interaction. The latter is strong enough to prevent them from crossing through each other on relevant simulation timescales. Thus, entanglements, once formed by motor rotation, are unable to dissipate by simple chain crossing. The motor unit consists of 6 beads connected as shown in Fig. 2c: the rotor and the stator both consist of 3 beads and are driven by a quadruplet of forces acting at beads A, B, C and D perpendicular to the motor axis $\mathrm{F}-\mathrm{D}$ as well as to the stator (CG-D) and the rotor axes (A-F-B). ${ }^{33}$ To ensure the rigid geometry of the motor, an additional angular stiffness term is added ensuring the orthogonality of the axis FG on the stator and rotor.

When acted upon the forces $f$, as shown in Fig. 2c, such a configuration is both net force-free and (external) torque free. And yet the device generates a relative internal torque $M=f b$ between its stator and rotor part. The mutual excluded volume interactions of the 6 motor beads are inactivated to prevent steric hindrance of the stator with rotor and to allow a free 

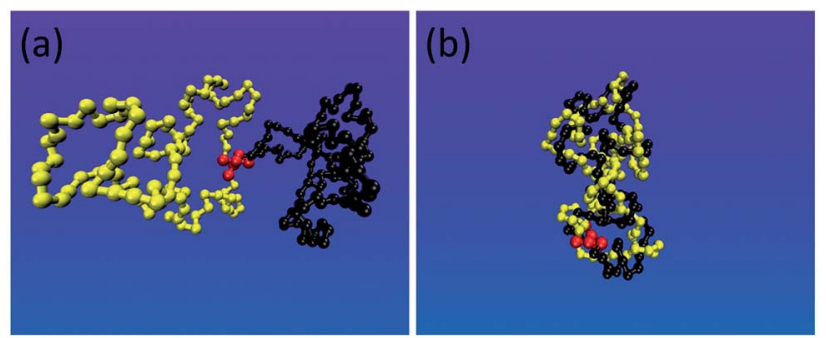

(c)
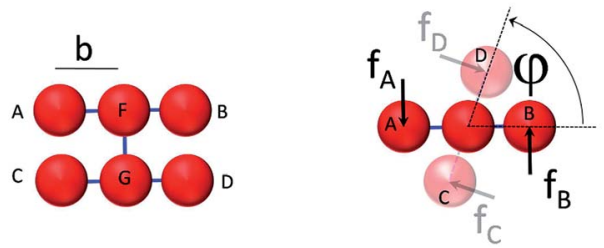

Fig. 2 (a) Molecular dynamics simulation of a single tanglotron. (b) Upon few rotations of the motor unit (red), the two polymer chains entangle and collapse significantly in size. (c) The implementation of the motor unit with 6 beads driven by a quadruplet of forces (right panel). The total force and torque vanish but the motor generates a relative torque doublet between its upper and lower sections.

motor rotation. The 6 rotor beads only interact with the chain particles to prevent accidental chain disentanglement.

The simulated tanglotrons with varying chain lengths of $N=$ 100, 200 and 400 particles per chain show an interesting trend, $c f$. Fig. 3. When actuated even with moderate torques $M$ of around $2 k T$, the conformation of the chains starts to change in a dramatic manner. Their radius of gyration $R_{\mathrm{g}}(M)$ consistently collapses from their initial unperturbed value $R_{\mathrm{g}}{ }^{0}$ to a plateau value $R_{\mathrm{g}}{ }^{\infty} \approx R_{\mathrm{g}}{ }^{0} / 2$. The threshold torque $M_{\text {crit }} \approx 2 k T$ leads to a strong chain entanglement which brings the chains closer together, leading to an effective compression of the tanglotron unit.

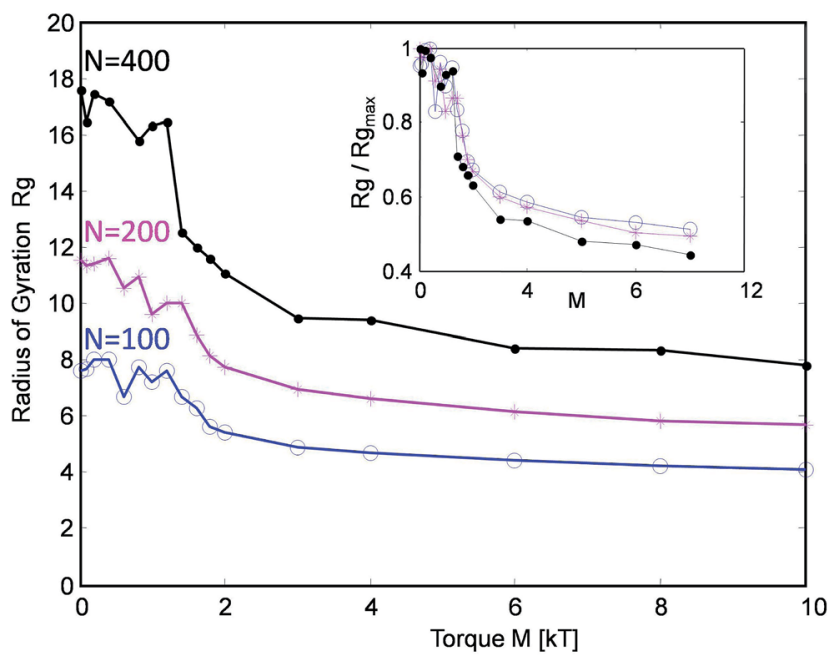

Fig. 3 The radius of gyration of the tanglotron particle as a function of the maximal torque generated by the motor for three different chain lengths.
The chain collapse is brought about by the increase of the mean motor angle $\langle\varphi(M)\rangle$ and the associated inter-chain linking number $\operatorname{Lk}(M)=-\frac{1}{2 \pi} \varphi(M)$, cf. Fig. 4. The latter grows with the increasing motor torque in an interesting manner. At small torques up to $M \approx 1 k T$, there is little or no change of Lk. At slightly larger torques up to about $5 k T$, the linking number starts to grow quickly. At larger values, it saturates to a plateau value $\mathrm{Lk}_{\max }$. The evolution of $\mathrm{Lk}$ for different chain lengths scales linearly with the maximal plateau value, which itself is proportional to the total chain length $N$, as further discussed below ( $c f$. also Fig. 7).

Interestingly, the chains collapse quickly to about $50 \%$ of their size even at low torques $M \approx 2 k T$ and one sees very little evolution in size beyond that. At the same time, the entanglement Lk (cf. Fig. 4) continues to grow up to $M \approx 10 k T$. This indicates that already in the weak entanglement regime the chains are strongly compacted on the large scale. Beyond that the process of compaction is more subtle, happens on smaller scales down to the monomer size, but reflects in $R_{\mathrm{g}}$ only very weakly.

\section{B. Stored free energy}

By inverting the $M v s$. $\operatorname{Lk}(M)$ relationship, we can extract the effective free energy of the system

$$
F(\langle\mathrm{Lk}\rangle)=\int_{0}^{\mathrm{Lk}} M(\langle\mathrm{Lk}\rangle) 2 \pi \mathrm{d}\langle\mathrm{Lk}\rangle
$$

This procedure is equivalent to performing a Legendre transform on the Gibbs potential $G(M)=-\int_{0}^{M} 2 \pi\left\langle\operatorname{Lk}\left(M^{\prime}\right)\right\rangle \mathrm{d} M^{\prime}$ and applies where the fluctuations of Lk are small or negligible. We cross-check it in the appendix against a direct measure of the free energy from the probability distribution of $\mathrm{Lk}$ for small Lk. The result plotted in Fig. 5 shows a remarkable behavior. Most notably, at small Lk the free energy is proportional to the

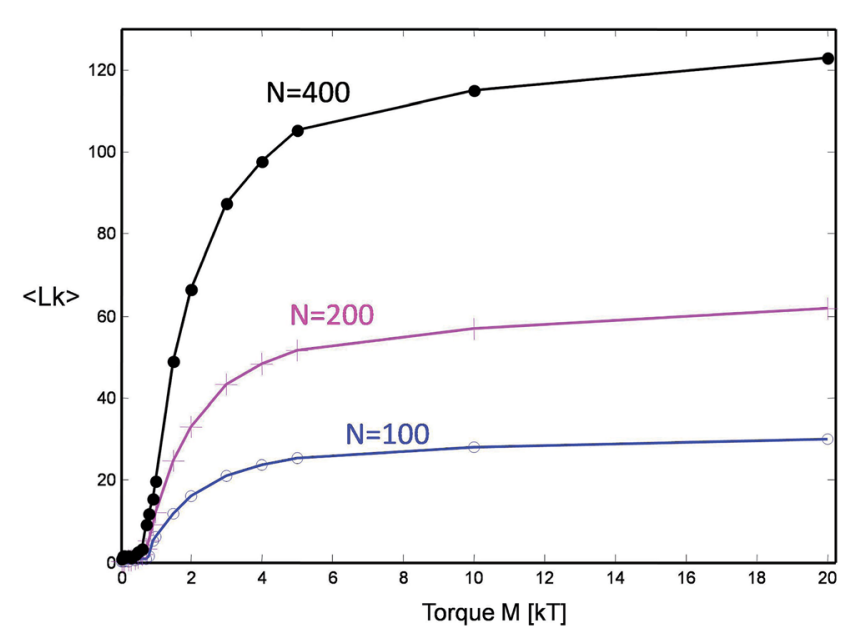

Fig. 4 The number of stored topological links (linking number Lk) for three chain lengths as a function of applied motor torque. 


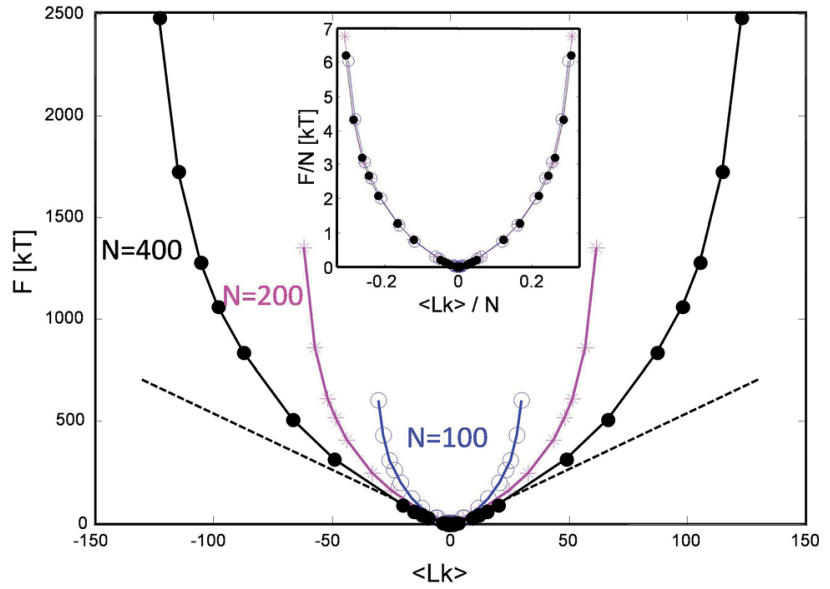

Fig. 5 The stored entanglement free energy as a function of the number of stored topological links/turns (linking number Lk) for three chain lengths.

number of links. Due to symmetry, the free energy is the same for the positive and negative linking numbers of the same magnitude. This symmetry and the linear (rather than a quadratic) growth slightly away from zero lead to an apparent non-analytic cusp of $F(\mathrm{Lk})$ at $\mathrm{Lk}=0$.

The result is reproducible and very similar for all chain lengths. What does this mean? The result indicates that at low linking number density, the topological links behave as discrete particle-like entities, with a certain chemical potential $\mu=F(1)$ for their injection. This leads to a formal cusp of the free-energy extrapolated towards $L k=0$. That observation agrees with the common polymer-physics intuition that, at low density, each entanglement costs a small amount of energy comparable to $k T$. This very reasonable assumption common in the literature ${ }^{13-15}$ comes from the fact that all free energies are entropic in nature and the only energy scale is the thermal one. However there could be a geometric prefactor to this energy scale. Indeed from the free energy slope at $\mathrm{Lk}=0$, we find $\mu \approx 6 k T$ i.e. each complete topological link (at low density) costs roughly that amount. This value of $\mu$ is compatible with the direct measure of the free energy at low link presented in the Appendix.

Interestingly, in the large Lk regime, after the initial linear growth, $F(\mathrm{Lk})$ begins to shoot up steeply. This behavior can be understood as densely packed links start to strongly interact with each other. Eventually the chains are expected to reach a densest topological packing state, corresponding to roughly $\approx 4$ beads per turn on each chain. This result is analogous to previous computer studies on the optimal packing of inflating torus knots $^{39}$ as well as mechanical 2 ply structures ${ }^{40}$ at vanishing temperature. There, as in our case, the geometry and the excluded volume of the chains prevent any further topological packing beyond the densest packing limit ( $c f$. below).

\section{Tanglotron's equation of state}

To understand deeper the high entanglement regime, which is most relevant for free-energy storage, let us take the simplest phenomenological approach and consider a tightly packed helix of radius $R$ and a pitch height $H$ shown in Fig. 6a.

The helix with the inclination angle $\alpha=\arctan \left(\frac{H}{2 \pi R}\right)$ has a length per turn $L_{\text {turn }}=\sqrt{(2 \pi R)^{2}+H^{2}}$ and the linking number per monomer is given by:

$$
\mathrm{Lk}=\frac{b}{L_{\mathrm{turn}}}= \pm \frac{b}{\sqrt{(2 \pi R)^{2}+H^{2}}}
$$

where the $\operatorname{sign} \pm=\operatorname{sign}(H)$ is positive/negative for a right/left handed helix, respectively.

In the following, we conveniently scale the radius and pitch $r=\frac{R}{b / 2}, h=\frac{H}{b / 2}$ by the actual radius of the monomer $b / 2$. In Fig. $6 \mathrm{~b}$, we have evaluated numerically the region of geometrically admissible helix pitches and radii satisfying the condition of mutual non-penetration of the two chains. We obtain that the state with maximum packing density (the bluest region) has $h=$ $h_{0} \approx 5.3$ and $r=r_{0} \approx 1.06$. The maximal linking number density resulting from these values is $\left|\mathrm{Lk}^{\max }\right| / N \approx 0.25$. This energy minimizing state would be ideally assumed for a perfect (double polymer) helix at zero temperature $T=0$ if fluctuations can be neglected.

However at any finite temperature, the mutual entropic confinement effects of the two polymer chains become the dominant effect, preventing further entanglement before the contact is established. In the limit of a tightly packed helical arrangement, the chain monomers start to dramatically loose their translational entropy. In particular, the motions in the radial direction of the helical arrangement become strongly prohibited by the geometric constraints. To model this behavior let us consider the twisting energy per monomer $E=E_{\text {tot }} / N$ that can be expressed as
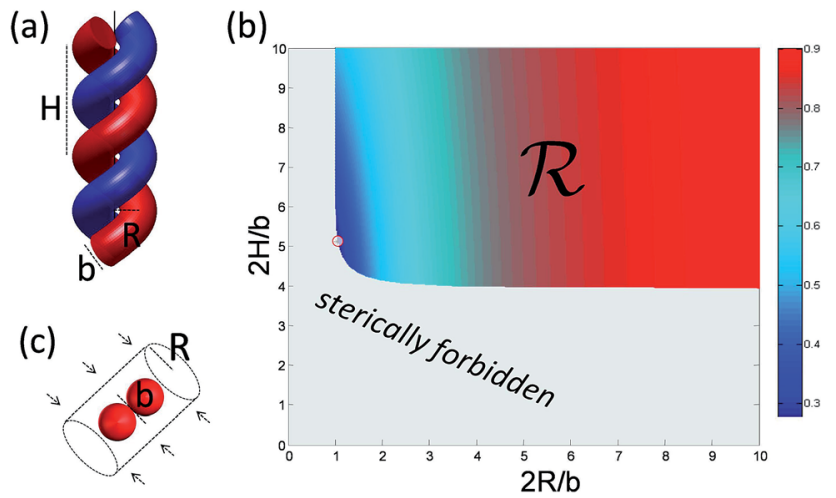

Fig. 6 The geometric model of high entanglement. (a) At high linking number densities, the two chains strongly confine each other. They form a tight helix with a pitch $H$ and radius $R$. (b) The sterically admissible helical pitches and radii superposed with the entanglement energy $M \varphi$ (color contour map), with the absolute minimum under this constraint at the region boundary (circle at the edge of the blue region). (c) The monomers of one chain are forced into an effective tube (set by the presence of the other chain) giving rise to entropic forces opposing the tube's collapse. 


$$
E=-2 \pi M \mathrm{Lk}
$$

To obtain the Gibbs potential $G(M)$ (Gibbs free energy), ideally, we should integrate the partition function over all possible configurations weighted according to their Lk. This procedure is not analytically tractable due to the subtle nature of the linking number which is a double integral over the two chains in the most general case. Instead we chose to integrate over all possible helices, whose Lk obeys a simple expression. This is physically justified as at high torque helices are indeed the preferred shapes.

In the limit of strong entanglement, each monomer starts to behave as an independent entity and we can approximate the Gibbs potential per monomer by

$$
G_{\mathrm{mon}}(M)=-k T \ln Z_{\mathrm{mon}}
$$

with the partition function of the monomer given by

$$
Z_{\text {mon }}(M)=\int_{\mathscr{R}} \exp \left(\frac{2 \beta M}{\sqrt{r^{2}+\left(\frac{h}{2 \pi}\right)^{2}}}\right) \mathrm{d} r \mathrm{~d} h
$$

with $\beta=1 / k T$. In the partition function, the integral goes over the admissible region $\mathscr{R}$ of the configurational space where the excluded volume constraint is satisfied ( $c f$. Fig. 6b). For large $M$, the main contribution to the partition function comes from a certain corner of the helix configurational space with the lowest energy $E(h, r)$ ( $c f$. circle in the blue region of Fig. 6b). There the partition function can be evaluated numerically, but it is conceptually more illuminating to estimate it by an approximate argument. For that we replace the exact boundary of the region $\mathscr{R}$ by its two asymptotes given by $r=1$ and $h=4$, corresponding to two extreme types of self-contacting helices with $R=b / 2, H \gg R$ and $H=2 b, H \ll R$, respectively. For large torques $\beta M \gg 1$, we expand the exponent in $Z_{\text {mon }}$ around these values using $h$ $=4+\delta h$ and $\mathrm{r}=1+\delta r$ with $\delta h$, and $\delta r \ll 1$. In this way, we easily obtain

$$
Z_{\text {mon }}(M) \propto(\beta M)^{-2} \mathrm{e}^{2 \pi \gamma \beta M}
$$

with the geometric packing constant $\gamma=1 / \sqrt{\pi^{2}+4} \approx 0.27$. The Gibbs potential $G(M)$ (Gibbs free energy) is then given by

$$
G_{\mathrm{mon}}(M) \approx-2 \pi M \gamma+2 k T \ln (M \beta)
$$

The mean linking number per monomer $\langle\mathrm{Lk}\rangle=-\frac{1}{2 \pi N} \frac{\partial G_{\mathrm{mon}}(M)}{\partial M}$ is then given by the elegant expression

$$
\langle\mathrm{Lk}\rangle=N\left(\gamma-\frac{k T}{\pi M}\right)
$$

The first term $\gamma$ is the maximal entanglement packing density while the second term is the "squeeze-out" of monomer's 2 degrees of freedom ( $H$ and $R$ ) by the applied torque.
In our simple approximate model $\gamma=\gamma_{\text {simple }}=0.27$ but in principle this value can be different, in fact larger, due to more optimal packing possible for two chains. If we allow for a slight adjustment of the $\gamma$ term due to such effects, we obtain very good agreement with the simulation data, $c f$. Fig. 7.

The somewhat higher packing density of $\gamma=0.32$ likely originates from a symmetry breaking along the helix similar to the one found by Pieranski for extremely knotted torus knots. ${ }^{39}$ Indeed for very large torques $M>10 \mathrm{kT}$, this type of dense zig-zag helix arrangement, where strand 1 first wraps around 2 one round, while 2 remains straight, and then they switch roles, can be also observed in our simulation snapshots, $c f$. Fig. $2 \mathrm{~b}$.

An approximate value of the free energy $F(\mathrm{Lk})$ which is a function of Lk (rather then applied torque) can be obtained by Legendre transform of the Gibbs potential and reads

$$
F(\mathrm{Lk})=-2 k T N \log \left(1-\frac{|\mathrm{Lk}|}{\gamma N}\right)
$$

where we chose $F(\mathrm{Lk}=0)=0 .{ }^{\mathbf{4 1}}$ Expanding the free-energy far from saturation, $|\mathrm{Lk}| \ll \gamma N$ yields $\mu=2 k T / \gamma$ which is consistent with the measured $\mu=6 k T$. One may wonder why the Legendre transform describes the data even at low torque $M \sim 1 k T$, despite possible Lk fluctuations which make this procedure only semi-quantitative. One reason may be found in the fact that while the shape fluctuations are strong, the relevant Lk fluctuations are less pronounced.

A simple estimate of the free energy density stored in the tanglotron (over a volume $R_{\mathrm{g}}{ }^{3}$ ) for torques $M$ of order of $10 \mathrm{kT}$, for the chain-lengths considered here $(N=100-400)$, leads to typical values between $100 \mathrm{MPa}$ and $1 \mathrm{GPa}$. The typical elastic moduli should be well beyond those of typical polymer gels but in the same range and comparable to those of proteins. Injecting entanglements even at moderate motor torques should therefore lead to a strong rigidification of the tanglotron's chains, comparable to those introduced in peptides by non-covalent bonds.

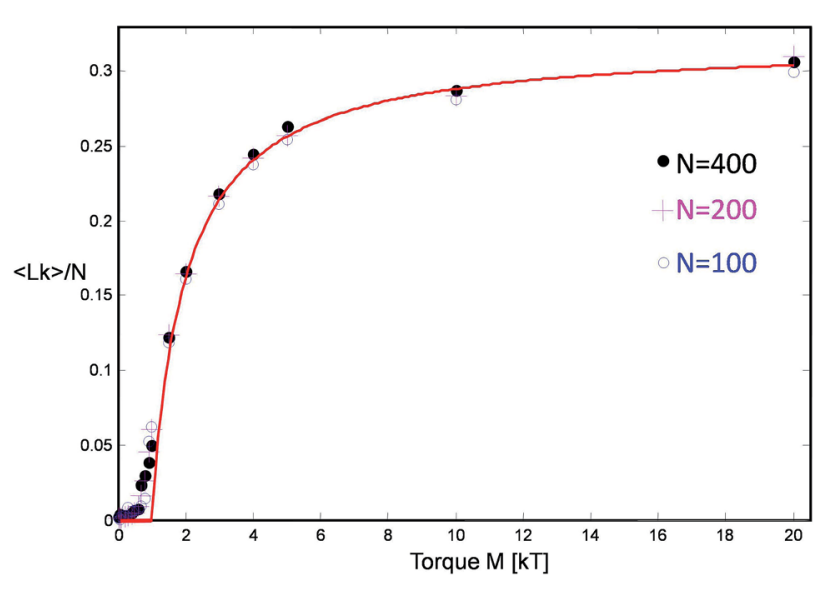

Fig. 7 The linking number Lk per unit length for three chain lengths as a function of applied motor torque and the comparison with the theoretical model (red curve) cf. eqn (8). 


\section{A. The scaling picture: the blob double helix}

The upper considerations in the high entanglement regime suggest a surprisingly simple picture of how the entanglement energy is stored for low entanglement densities. If we look back to the inset of Fig. 5, where the entanglement density (per monomer) and free energy per monomer are plotted for three chain lengths, we can make an interesting observation. For all entanglement densities, the energy densities collapse onto a single curve. That is, the monomers seem to behave on average as identical entities with the same energy content per monomer, with no signs of explicit system size dependence. The corresponding free energy is extensive in the system size $N$.

This is an indication that we could make a simple blobargument even for a small entanglement case by replacing what were single beads with size $b$ in the high-entanglement limit by blobs of size $l>b$ carrying each $\sim k T$ of energy. A chain of such $l$ - blobs is now packed in such a manner to mimic the previous case of the densest helical packing of single beads in the highentanglement limit. To inject a single entanglement, we need then roughly $\sim 3-4$ blobs per chain, which amounts to a free energy cost of $6-8 k T$ per single entanglement. This expectation is in surprisingly close agreement with the chemical potential $\mu$ $\approx 6 k T$ per entanglement estimated from the simulations. This suggests that the simple "blob-helix" model predicts the behavior of the free energy even for moderate entanglement.

The observation that at intermediate densities a single entanglement costs a fixed amount of energy deserves a few comments. Note that a similar behavior of the free energy as a function of Lk, $F(\mathrm{Lk}) \propto|\mathrm{Lk}|$, has been observed by Marko ${ }^{22}$ for two entangling mutually pinned DNA rings with excluded volume. A related study by Walter et al. ${ }^{21}$ on flexible chains (with excluded volume) wrapping around a bar also finds a transition from a Gaussian $F(\mathrm{Lk}) \propto \mathrm{Lk}^{2}$ scaling to a weaker power law $F(\mathrm{Lk})$ $\propto|\mathrm{Lk}|^{\alpha}$ with $\alpha$ not far from unity and with a typical cross-over occurring at $\mathrm{Lk} \sim \ln N$. In our current case, such Gaussian behavior is difficult to observe, as it is masked by another effect: the mutual entropic hindrance of the two chains at the motor junction for $\mathrm{Lk}=0$. The latter gives rise to an inverted quadratic behavior around 0 and to additional shallow minima in the free energy, $c f$. Fig. 9 in the Appendix. Furthermore, it is interesting to note that the constant free energy per entanglement found by Marko of 3.6kT per entanglement is comparable to, yet roughly two times smaller than the $\mu \approx 6 k T$ we find here. The reason can be found in the fact that Marko's Monte Carlo study measures the contribution of arbitrary complex catenanes while in our case only toroidal inter-chain braids are generated by the nature of motor unit rotation. Restricting the type of entanglement (from arbitrary to toroidal ones) to a subset obviously increases the free energy per entanglement in agreement with the larger value we find.

\section{Discussion}

In this report, we have considered the energy storage capacity and configurational properties of a rotary motor unit self-connected with two entangling polymer chains - the "tanglotron" device. The tanglotron principle is scalable and rather flexible. Several motor candidates, including artificial ones like the Feringa rotary engine $^{\mathbf{1 0}}$ (driven by light) or biological ones like ATP synthase ${ }^{4}$ (supplied with excess ATP), can be utilized.

The basic energy "currency" that we propose here for storage and transmission is the topological link. This entity has a number of interesting and useful properties including:

(1) A fine grained and tunable elementary "quantum" of (free) energy. For a low link density, we expect a few kTs per entanglement.

(2) For higher densities, the links strongly interact which increases their energy to larger values. This might be advantageous in some situations where larger free energies per turn are required.

(3) The topological links are easy to store and can be specifically transported to the location where they can be utilized, i.e. to a free energy consumer. They directly interact with the mechanical degrees of freedom of the consumer, but being topological rather than strict geometric entities, they do so without a strong dependence on the environmental constraints.

(4) The topo-links are stable, long lived topological objects. They do not simply vanish into the solution but rather remain localized in the device. Therefore they can intermittently store and buffer the mechanical work produced by a motor and become utilized at a later time point by a consumer. In this sense, they can act as a "time-scale bridge" or "time-gear" bridging the otherwise vastly different conformational relaxation timescales of the motor and the consumer.

(5) The energy of the entanglements can be additionally further tuned under appropriate solvent conditions. It can be increased by charging the chains in an identical manner (positive or negative) or can be decreased by increasing the chains' affinity as compared to the solvent, i.e. making them less soluble.

\section{Outlook}

A rather non-exhaustive list of potential tanglotron related applications, giving us a flavor of its utility is outlined in Fig. 8ad. Examples include the following aspects which are interesting to mention:

Application 1 (artificial muscle material, see Fig. 8a): an obvious generalization consisting of tanglotrons cross-linked in a fully reticulated gel - the "topo-gel" deserves separate consideration. However it is intuitively clear that upon motor operation such a gel will collapse by the same order of magnitude as the single "topo-core" (tanglotron) particle, namely around 50 percent in each spatial direction. Both in the topo-gel and in the topo-core particle, the particular arrangement of the stator-rotor of the motor unit and its connectivity established by the polymer chains ensure that the construct acts as a topomechanical battery.

Notably, by the action of one small fraction of the whole system mass (the rotary motors) acting as an "enzymatic" element, the system (the polymer gel) is progressively converted, via the process of topological "charging”, into an energy 
(a)

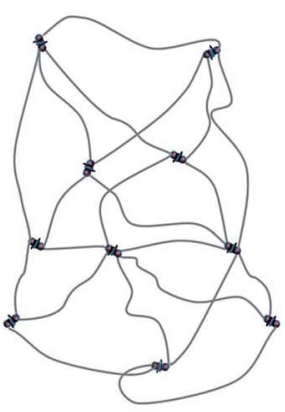

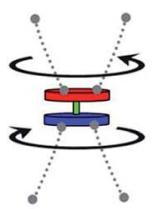

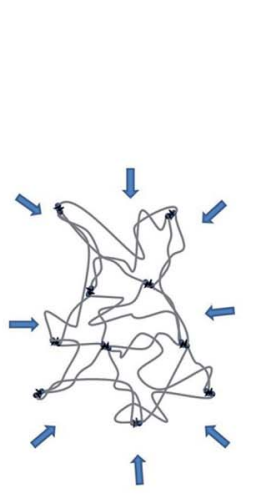

(c)

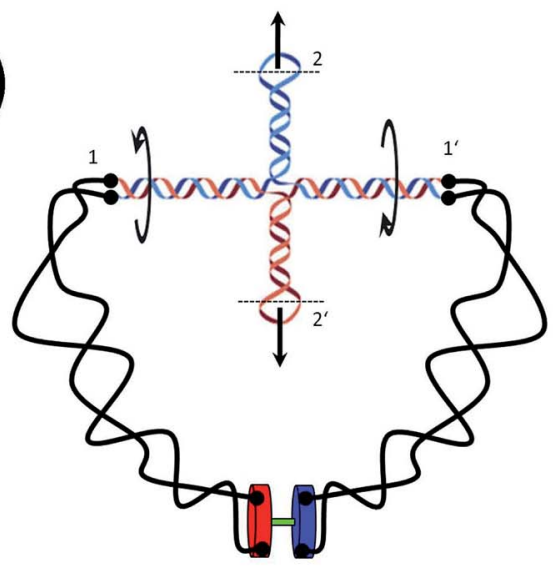

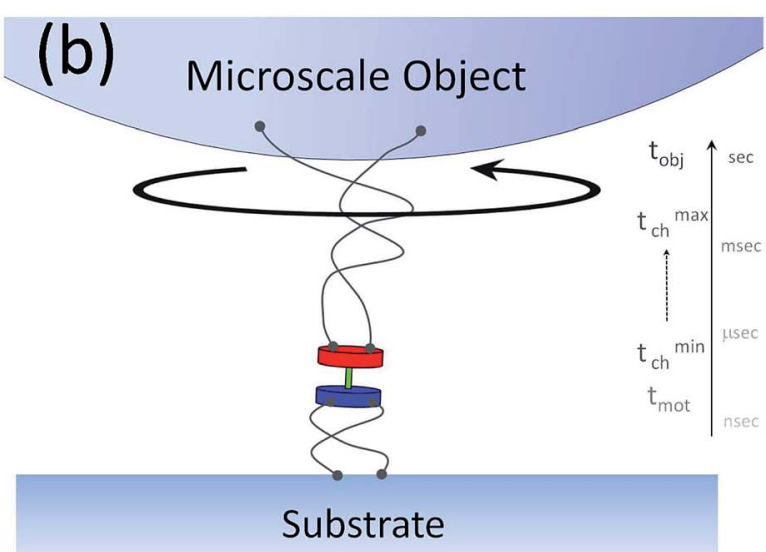

(d)

Substrate

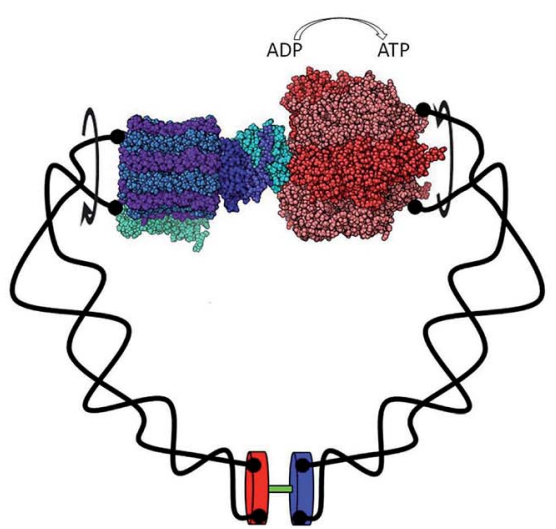

Fig. 8 Applications of the tanglotron. (a) A "topo gel" consisting of reticulated tanglotrons acts as an artificial muscle. (b) The time-scale-bridging function of a tanglotron. It can easily move objects billion times heavier than itself via the trick of transient energy buffering in the chains. (c) The rotary motion can be transformed into linear actuation via a DNA cruciform structure (at positions 1 and $1^{\prime}$ ). The tanglotron device can now bridge to any DNA nano-assembly if we cut/open the end loops of the cruciform at connections 2 and $2^{\prime}$. The cruciform transmits both rotary to rotary motion and rotary to linear motion. (d) The motor unit can be directly coupled to distant catalytic centers that act as energy consumers, here an ATP-synthase. The (transmembrane) proton motive subunit of the ATP-synthase is replaced by the distant rotary motor, softly coupled by polymer chains. In reverse, supplying the synthase with excess ATP turns it itself a rotary motor unit.

enriched contracted structure. Such a gel would operate as an artificial muscle, although only in one direction (contraction). To make the contraction reversible, a second passive topologydissipating unit could be inserted to counteract the action of the motor unit.

Application 2 (motion amplification, see Fig. 8b): a tanglotron can move objects billions of times heavier than itself. The fast elementary stepping of the motor unit, which occurs typically in nanoseconds to microseconds, would be dramatically suppressed by any rigid coupling to a much bigger object, like the slowly rotating macroscopic bead shown in Fig. 3. However, the situation is much more hopeful when the motor is coupled to the "consumer" through the two soft polymer chains. The latter have a broad distribution of relaxation timescales, with the shortest ones typically overlapping with the motor stepping timescales. The topological links injected by the rotor are transiently buffered in the polymer chains to be utilized in the bead rotation on much longer time-scales (seconds).
Application 3 (linear actuation and DNA interfacing, see Fig. 8c): the rotary motion can be simply transformed into linear actuation. This can happen e.g. via a DNA cruciform structure coupled to the polymer chains. In this way, the tanglotron device can principally bridge to any DNA nano-assembly via the end loops of the cruciform at connections 2 and $2^{\prime}$. The DNA nano-assembly realm ${ }^{34-38}$ is a very promising target and a possible "killer-application" for the tanglotron device.

Application 4 (mechanochemical coupling to reactions, see Fig. 8d): the tanglotrons should also optimally allow the release of the stored stress by coupling the dissipation of the entanglements to a chemical reaction. This can be done also in a useful manner. The motor unit can be directly coupled to "energy consumers", like for instance distant catalytic centers. One curious, but feasible consumer would for instance be an ATP-synthase - the cellular enzyme synthesizing ATP from the proton-motive force across cellular membranes. The transmembrane proton-drive subunit of the ATP-synthase can be replaced by a tanglotron, i.e. a distant rotary motor, softly 


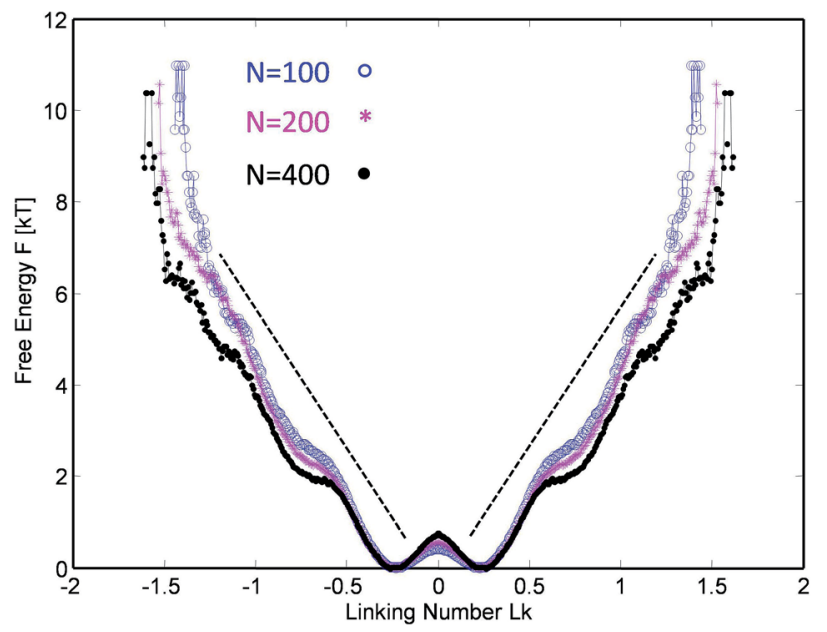

Fig. 9 The stored entanglement free energy determined from the histogram statistics, as a function of the number of stored topological links (linking number Lk) for three chain lengths. The dashed slopes correspond to $6 k T$ per turn.

coupled by polymer chains. Interestingly, in the reverse direction, supplying the synthase with excess ATP makes it itself a rotary motor unit which can be used as an ATP-driven rotor drive of the tanglotron unit.

\section{Conclusions}

A tanglotron ( $c f$. Fig. 1) is a molecular device that converts repetitive cyclic motion into polymer self-entanglement. As a consequence, it induces a large polymer contraction and stores topo-mechanical energy in this process. The tanglotron's chains can act as a practical energy conversion and storage unit as well as a long range energy transmission medium. The tanglotron can be seen as an active topological nanoscale gear that acts as a soft and "geometry insensitive" nano-mechanical element able to adapt to any relative reorientation and repositioning of the motor and "consumer". What is transmitted are topological entanglements, entities which are robust under geometric transformations. An effective transmission of rotation from a (fast) molecular engine device to a (slow) "consumer" element can only work if there is some form of intermediate mechanical energy storage, i.e. an efficient buffering of energy. This task can be practically fulfilled by the topological entanglement unit of the tanglotron.

It will be the central future challenge to synthesize realizations of tanglotrons, to characterize the chemical, thermodynamical and mechanical properties of such novel molecular devices and explore their utility as universal driving components of future smart materials.

\section{Appendix: the free energy at zero torque}

It is interesting to reinvestigate the wedge-like behavior of the free energy by another method that allows us to further zoom into the region around $\mathrm{Lk}=0$. At vanishing torque, i.e. when the motor is free to rotate, the probability density $p(\varphi)$ of the motor angle $\varphi$ (i.e. its histogram of values) can be used to extract the free energy from

$$
F(\mathrm{Lk})=-k T \ln p(\mathrm{Lk})+\text { const. }
$$

The result plotted in Fig. 9 shows similar wedge like behavior around $\mathrm{Lk}=0$ as shown in Fig. 5. However it displays some more interesting fine details. In particular, it shows that the naively expected orientation $\varphi=0$ is not preferable. Instead, there are two close symmetric minima at around $\varphi \approx \pm \pi / 2$, induced by the mutual entropic hindrance of the two polymer chains coming out from the motor unit. At $\varphi=0$, the chains would emerge very close to each other giving rise to mutual entropic forces, thus pushing the preferred motor angle slightly away from 0 . At larger values $\operatorname{Lk} \sim 1(\varphi \sim 2 \pi)$, the free energy grows linearly, with a slight modulation due to inter-chain steric hindrance, displaying a similar slope of $\sim 6 k T$ per turn found in the main text.

\section{Acknowledgements}

The authors thank Quan Li, Gad Fuks, Hendrik Meyer, Nicolas Giuseppone, Joachim Wittmer, Justin Foy, and Falko Ziebert for fruitful discussions.

\section{References}

1 R. Dawkins, Why don't animals have wheels?, Sunday Times, November 24, (1996).

2 I. M. Kulić, R. Thaokar and H. Schiessel, Twirling DNA rings - Swimming nanomotors ready for a kickstart, Europhys. Lett., 2005, 72, 527.

3 V. Balzani, A. Credi and M. Venturi, Molecular Devices and Machines, Wiley-VCH, 2003.

4 J. M. Berg, J. L. Tymoczko and L. Stryer, Biochemistry, W. H. Freeman, 5th edn, 2002.

5 H. Noji, R. Yasuda, M. Yoshida and K. Kinosita, Direct observation of the rotation of F1-ATPase, Nature, 1997, 386, 299-302.

6 H. C. Berg and R. A. Anderson, bacteria swim by rotating their flagellar filaments, Nature, 1973, 245, 380.

7 A. Maxwell and A. D. Bates, DNA topology, Oxford University Press, 2005.

8 C. Mao, W. Sun, Z. Shen and N. C. Seeman, A nanomechanical device based on the $\mathrm{B}-\mathrm{Z}$ transition of DNA, Nature, 1999, 397, 144-146.

9 H. Yan, X. Zhang, Z. Shen and N. C. Seeman, A Robust DNA Mechanical Device Controlled by Hybridization Topology, Nature, 2002, 415, 62-65.

10 N. Koumura, R. W. J. Zijlstra, R. A. van Delden, N. Harada and B. L. Feringa, Light-driven monodirectional molecular rotor, Nature, 1999, 401, 152-155.

11 T. R. Kelly, H. De Silva and R. A. Silva, Unidirectional rotary motion in a molecular system, Nature, 1999, 401, 150. 
12 J.-M. Lehn, Conjecture: Imines as Unidirectional Photodriven Molecular Motors-Motional and Constitutional Dynamic Devices, Chem.-Eur. J., 2006, 12, 5910-5915.

13 P. G. de Gennes, Scaling concepts in polymer physics, Cornell University Press, Ithaca, 1979.

14 P. G. De Gennes, Entangled polymers, Phys. Today, 1983, 36(6), 33-31.

15 M. Doi and S. Edwards, The Theory of Polymer Dynamics, Clarendon Press, 1999.

16 F. Spitzer, Some Theorems Concerning 2-Dimensional Brownian Motion, Trans. Amer. Math. Soc., 1958, 87, 187197.

17 S. Prager and H. L. Frisch, Statistical Mechanics of a Simple Entanglement, J. Chem. Phys., 1967, 46, 1475.

18 S. F. Edwards, Statistical mechanics with topological constraints: I, Proc. Phys. Soc., 1967, 91, 513.

19 J. Rudnick and Y. Hu, The winding angle distribution of an ordinary random walk, J. Phys. A: Math. Gen., 1987, 20, 44214438.

20 A. Grosberg and H. Frisch, Winding angle distribution for planar random walk, polymer ring entangled with an obstacle, and all that: Spitzer-Edwards-Prager-Frisch model revisited, J. Phys. A, 2003, 36, 8955.

21 C. Walter, G. T. Barkema and E. Carlon, The equilibrium winding angle of a polymer around a bar, J. Stat. Mech.: Theory Exp., 2011, P10020.

22 J. F. Marko, Linking topology of tethered polymer rings with applications to chromosome segregation and estimation of the knotting length, Phys. Rev. E, 2009, 79, 051905.

23 T. R. Strick, J.-F. Allemand, D. Bensimon, A. Bensimon and V. Croquette, The Elasticity of a Single Supercoiled DNA Molecule, Science, 1996, 29, 1835.

24 J. F. Marko and E. D. Siggia, Fluctuations and supercoiling of DNA, Science, 1994, 265, 506; J. F. Marko and E. D. Siggia, Statistical mechanics of supercoiled DNA, Phys. Rev. E, 1995, 52, 2912.

25 J.-C. Walter, M. Baiesi, E. Carlon and H. Schiessel, Unwinding Dynamics of a Helically Wrapped Polymer, Macromolecules, 2014, 47, 4840.

26 J. F. Marko, Supercoiled and braided DNA under tension, Phys. Rev. E, 1997, 55, 1758.

27 R. C. Ball, M. Doi, S. F. Edwards and M. Warner, Elasticity of entangled networks, Polymer, 1981, 22, 1010-1018; P. G. de Gennes, Sliding gels, Physica A, 1999, 271, 231-237.

28 A. Harada and M. Kamachi, Complex formation between poly(ethylene glycol) and a-cyclodextrin, Macromolecules, 1990, 23, 2821-2823; Y. Okumura and K. Ito, The polyrotaxane gel: a topological gel by figure-of-eight crosslinks, Adv. Mater., 2001, 13, 485-487.
29 T. C. Boles, J. H. White and N. R. Cozzarelli, Structure of plectonemically supercoiled DNA, J. Mol. Biol, 1990, 213(4), 931-951.

30 N. R. Cozzarelli, T. Christian-Boles, and J. H. White, Primer on the topology and geometry of DNA supercoiling, DNA Topology and its Biological Effects, 1990, pp. 139-184.

31 T. Sanchez, I. M. Kulić and Z. Dogic, Circularization, Photomechanical Switching, and a Supercoiling Transition of Actin Filaments, Phys. Rev. Lett., 2010, 104, 098103.

32 I. M. Kulić, Evaluating polynomials on the molecular level - a novel approach to molecular computers, Biosystems, 1998, 45, 45.

33 The "rotor" and "stator" are identical and can be seen interchangably, as none of them is static and there is only relative motion between them induced by $M$.

34 N. C. Seeman, Nanotechnology and the double helix, Sci. Am., 2004, 290(6), 64-75.

35 W. K. Paul, Rothemund, Folding DNA to create nanoscale shapes and patterns, Nature, 2006, 440, 297-302.

36 F. C. Simmel and Y. Krishnan, Nucleic Acid Based Molecular Devices, Angew. Chem., Int. Ed., 2011, 50, 3124.

37 S. M. Douglas, H. Dietz, T. Liedl, B. Hogberg, F. Graf and W. M. Shih, Self-assembly of DNA into nanoscale threedimensional shapes, Nature, 2009, 459, 414; H. Dietz, S. M. Douglas and W. M. Shih, Folding DNA into Twisted and Curved Nanoscale Shapes, Science, 2009, 325, 725.

38 B. Yurke, A. J. Turberfield, A. P. Mills, F. C. Simmel and J. L. Neumann, A DNA-fuelled molecular machine made of DNA, Nature, 2000, 406, 605; S. F. Wickham, J. Bath, Y. Katsuda, M. Endo, K. Hidaka, H. Sugiyama and A. J. Turberfield, A DNA-based molecular motor that can navigate a network of tracks, Nat. Nanotechnol., 2012, 7, 169.

39 P. Pieranski, In search of ideal knots. In: Ideal Knots, Series on Knots and Everything, ed. A. Stasiak, V. Katritch and L. H. Kauffman, World Scientific, Singapore, 1998, vol. 19, pp. 20-41.

40 S. Neukirch and G. H. M. Van der Heijden, Geometry and Mechanics of Uniformn-Plies: from Engineering Ropes to Biological Filaments, J. Elastoplast., 2002, 69, 41-72.

41 Note that our calculations in this section are specialized to high link densities and describe the simulation results accurately at high torques, above the inflection point in the Lk vs. $M$ curve (Fig. 7), for $M>1.5 k T$. At low torques, the helix parameters show large fluctuations and the polymer connectivity becomes an issue. Nevertheless, the theoretical curve represented in Fig. 7 and complemented by zero response below $M=k T /(\pi \gamma) \approx k T$ nonetheless fairly accounts for the simulated equation of state. 